\title{
Note: A new truncation correction for the configurational temperature extends its applicability to interaction potentials with a discontinuous force
}

Cite as: J. Chem. Phys. 144, 056101 (2016); https://doi.org/10.1063/1.4941453

Submitted: 23 December 2015 . Accepted: 21 January 2016 . Published Online: 03 February 2016

Anders Lervik (D), Øivind Wilhelmsen, Thuat T. Trinh, and Edgar M. Blokhuis (D)
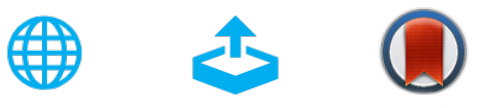

\section{ARTICLES YOU MAY BE INTERESTED IN}

Finite-size and truncation effects for microscopic expressions for the temperature at equilibrium and nonequilibrium

The Journal of Chemical Physics 143, 114106 (2015); https://doi.org/10.1063/1.4930540

\section{Equation of State for the Lennard-Jones Fluid}

Journal of Physical and Chemical Reference Data 45, 023101 (2016); https://

doi.org/10.1063/1.4945000

Development of DPD coarse-grained models: From bulk to interfacial properties

The Journal of Chemical Physics 145, 054107 (2016); https://doi.org/10.1063/1.4960114

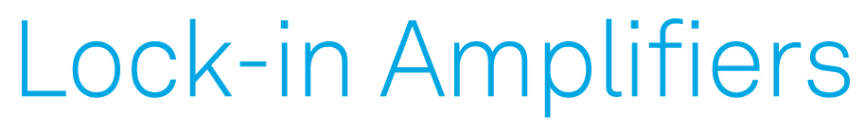

... and more, from DC to $600 \mathrm{MHz}$ Watch 


\title{
Note: A new truncation correction for the configurational temperature extends its applicability to interaction potentials with a discontinuous force
}

\author{
Anders Lervik, ${ }^{1}$ Øivind Wilhelmsen, ${ }^{2, a)}$ Thuat T. Trinh, ${ }^{1}$ and Edgar M. Blokhuis ${ }^{3}$ \\ ${ }^{1}$ Department of Chemistry, Norwegian University of Science and Technology, NO-7491 Trondheim, Norway \\ ${ }^{2}$ SINTEF Energy Research, $N$-7465 Trondheim, Norway \\ ${ }^{3}$ Colloid and Interface Science, Gorlaeus Laboratories, Leiden Institute of Chemistry, P.O. Box 9502, \\ 2300 RA Leiden, The Netherlands
}

(Received 23 December 2015; accepted 21 January 2016; published online 3 February 2016)

[http://dx.doi.org/10.1063/1.4941453]

The configurational temperature has emerged as a useful tool to compute the temperature in molecular simulations. ${ }^{1}$ It has been employed in Monte Carlo simulations as a diagnostic tool $^{2}$ and in molecular dynamics (MD) simulations where it has given enhanced understanding of systems in Poiseuille flow, ${ }^{3-5}$ nanopores, ${ }^{6}$ as well as many other systems. ${ }^{7-12}$ Jepps et $a l^{4}{ }^{4}$ showed that the configurational temperature can be determined from

$$
k_{\mathrm{B}} T_{\mathrm{con}}=\frac{\left\langle\sum_{i=1}^{N} \mathbf{F}_{i}^{2}\right\rangle}{\left\langle-\sum_{i=1}^{N} \nabla_{i} \cdot \mathbf{F}_{i}\right\rangle},
$$

where $k_{\mathrm{B}}$ is Boltzmann's constant, $T_{\mathrm{con}}$ is the configurational temperature, $\mathbf{F}_{i}$ is the force acting on particle $i, N$ is the total number of particles, and $\langle\cdot\rangle$ is the ensemble average. For spherically symmetric interaction potentials, statistical mechanics gives that ${ }^{14}$

$$
\begin{gathered}
\left\langle\sum_{i=1}^{N} \mathbf{F}_{i}^{2}\right\rangle=4 \pi \rho N \int_{0}^{\infty} \mathrm{d} r r^{2} g(r)\left[U^{\prime}(r)\right]^{2}, \\
\left\langle-\sum_{i=1}^{N} \nabla_{i} \cdot \mathbf{F}_{i}\right\rangle=4 \pi \rho N \int_{0}^{\infty} \mathrm{d} r r^{2} g(r)\left[U^{\prime \prime}(r)+\frac{2}{r} U^{\prime}(r)\right],
\end{gathered}
$$

where $\rho$ is the number density, $U(r)$ is the interaction potential, $r$ is the distance between the particles, and $g(r)$ is the radial distribution function. In the derivation of the expression for the configurational temperature in Eq. (1) presented by Jepps et al. ${ }^{4}$ the interaction potential is required to be continuously differentiable. Hence, the expression for $T_{\text {con }}$ is expected to be inaccurate for truncated (and shifted) potentials. No such truncation error is expected for the kinetic temperature since it uses only the particle momenta.

In the following, we consider a truncated and shifted Lennard-Jones (LJ) potential that is cut off at $r=r_{\mathrm{c}}$. When the configurational temperature is obtained by Eq. (1), with $\mathbf{F}_{i}$ set equal to zero beyond $r_{\mathrm{c}}$, we obtain a temperature which lies below the kinetic temperature as shown by the blue circles in Fig. 1. Previously, it was shown that this discrepancy can be partially resolved by adding tail corrections to the numerator $\left(\Delta_{n}\right)$ and denominator $\left(\Delta_{d}\right)$ in Eq. $(1)^{13}$

$$
\begin{aligned}
& \Delta_{n}=4 \pi \rho N \int_{r_{c}}^{\infty} \mathrm{d} r r^{2} g(r)\left[U^{\prime}(r)\right]^{2}, \\
& \Delta_{d}=4 \pi \rho N \int_{r_{c}}^{\infty} \mathrm{d} r r^{2} g(r)\left[U^{\prime \prime}(r)+\frac{2}{r} U^{\prime}(r)\right],
\end{aligned}
$$

where $U(r)$ represents the full $\mathrm{LJ}$ potential. Using $g(r)$ from Ref. 15, these corrections lead to the green squares in Fig. 1. The figure shows that Eq. (1) with the tail corrections in Eqs. (4) and (5) recovers the kinetic temperature of the system accurately, except at low truncation values and high densities (see Figs. 1(b)-1(d)). The rationale for using Eqs. (4) and (5) to extrapolate to the full potential is that the corrections keep the system temperature unchanged and that Eq. (1) is strictly valid for the full potential since the full potential is continuously differentiable. In this Note, our goal is to develop an alternative correction method that resolves the discrepancy between the kinetic and configurational temperature also for low truncation values and high densities.

To achieve this, we consider an interaction potential, $U_{\varepsilon}(r)$, which is smooth and differentiable, but which depends on some small parameter $\varepsilon$ in such a way that in the limit $\varepsilon \rightarrow 0$ it reproduces the cutoff and shifted LJ potential exactly. For any arbitrarily small but positive value for $\varepsilon$, Eq. (1) remains strictly valid and can be used. Then, in the limit $\varepsilon \rightarrow 0$, the first derivative of the interaction potential approaches

$$
U_{\varepsilon}^{\prime}(r) \longrightarrow U^{\prime}(r) \Theta\left(r_{\mathrm{c}}-r\right)
$$

where $\Theta($.$) is the Heaviside function. In the same limit, the$ second derivative of the interaction potential becomes more and more sharply peaked at $r=r_{\mathrm{c}}$, approaching

$$
U_{\varepsilon}^{\prime \prime}(r) \longrightarrow U^{\prime \prime}(r) \Theta\left(r_{\mathrm{c}}-r\right)-U^{\prime}\left(r_{\mathrm{c}}\right) \delta\left(r-r_{\mathrm{c}}\right)
$$



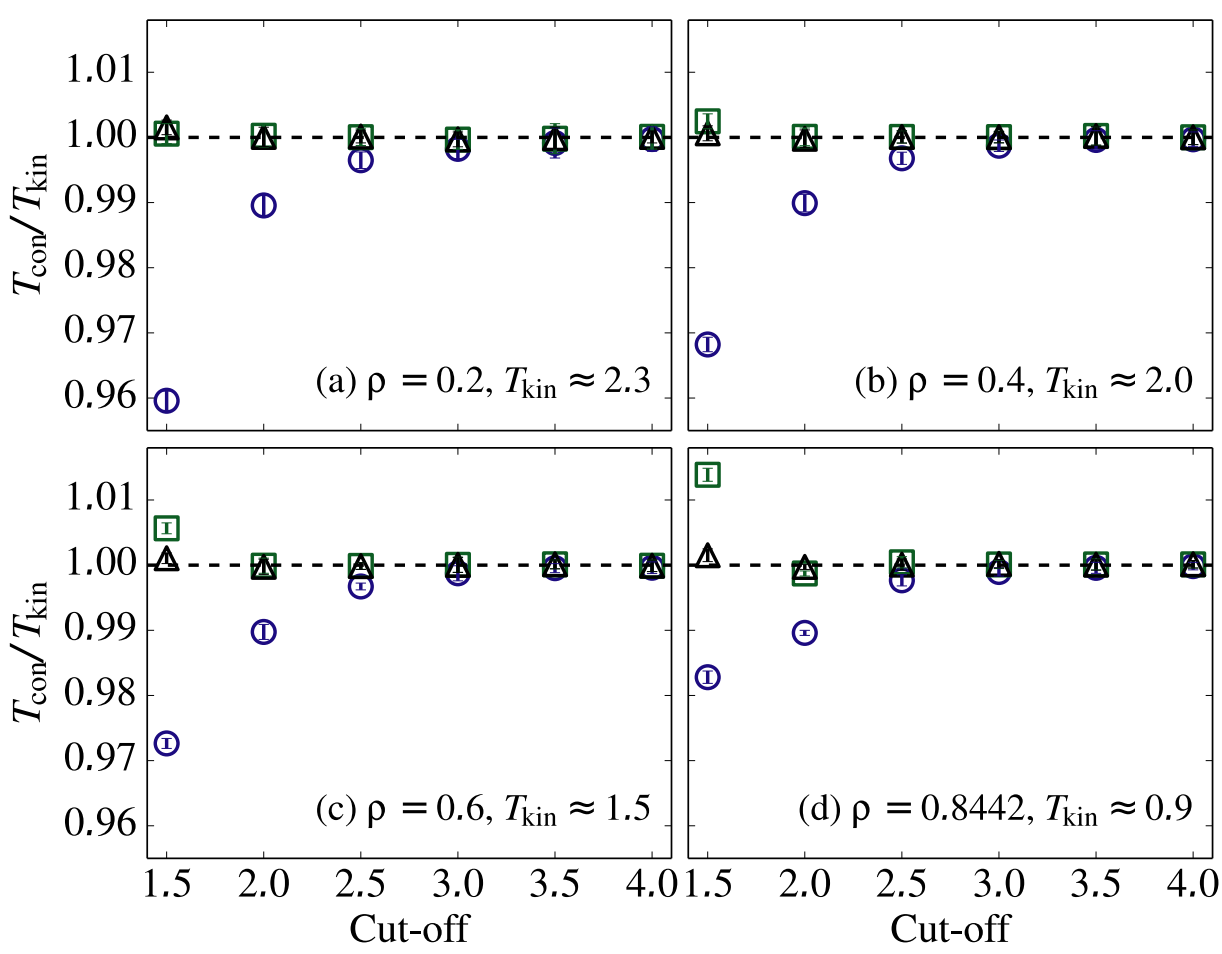

The result is that the expressions in Eqs. (2) and (3) continuously approach the limits

$$
\begin{aligned}
\left\langle\sum_{i=1}^{N} \mathbf{F}_{i}^{2}\right\rangle & \longrightarrow 4 \pi \rho N \int_{0}^{r_{\mathrm{c}}} \mathrm{d} r r^{2} g(r)\left[U^{\prime}(r)\right]^{2}, \\
\left\langle-\sum_{i=1}^{N} \nabla_{i} \cdot \mathbf{F}_{i}\right\rangle & \longrightarrow 4 \pi \rho N \int_{0}^{r_{\mathrm{c}}} \mathrm{d} r r^{2} g(r)\left[U^{\prime \prime}(r)+\frac{2}{r} U^{\prime}(r)\right] \\
& +\Delta_{\mathrm{disc}},
\end{aligned}
$$

where

$$
\Delta_{\text {disc }}=-4 \pi \rho N r_{\mathrm{c}}^{2} g\left(r_{\mathrm{c}}\right) U^{\prime}\left(r_{\mathrm{c}}\right) .
$$

Using $g(r)$ from Ref. 15 and the above correction, $\Delta_{\text {disc }}$, in the denominator of Eq. (1), we obtain the black triangles in Fig. 1. The figure shows that Eq. (1) with the new correction reproduces the kinetic temperature within the accuracy of the simulations for all the cases considered. Contrary to the tail corrections in Eqs. (4) and (5), Eq. (10) works also at low truncation values and high densities. As an approximation, one could set $g\left(r_{\mathrm{c}}\right)=1$, which gives for the LJ potential: $\Delta_{\text {disc }} \approx 96 \pi \rho N\left[2 r_{\mathrm{c}}^{-11}-r_{\mathrm{c}}^{-5}\right]$. (d) $\rho=0.8442, T_{\text {kin }} \approx 0.9$

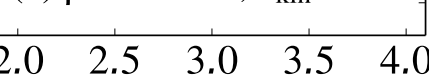

Cut-off
FIG. 1. The configurational temperature $\left(T_{\text {con }}\right)$ relative to the kinetic temperature $\left(T_{\text {kin }}\right)$ as a function of the cut-off distance and number density $(\rho)$ for the truncated and shifted LJ potential: uncorrected (blue circles), corrected with Eqs. (4) and (5) (green squares), and corrected with Eq. (10) (black triangles). The system contains 500 particles and the configurational temperature was calculated as explained in Ref. 13, except that we reduced the time-step with a factor of 2 for the smallest cut-offs (1.5 and 2.0) to avoid energy-drift.
To conclude, the success of the new correction in recovering the kinetic temperature of the system suggests that the expression for the configurational temperature given in Eq. (1) is valid also for interaction potentials with a discontinuous force, provided that the discontinuity is explicitly accounted for by the expression in Eq. (10).

${ }^{1}$ J. G. Powles, G. Rickayzen, and D. M. Heyes, Mol. Phys. 103, 1361 (2005). ${ }^{2}$ B. D. Butler, G. Ayton, O. G. Jepps, and D. J. Evans, J. Chem. Phys. 109, 6519 (1998).

${ }^{3}$ G. Ayton, O. G. Jepps, and D. J. Evans, Mol. Phys. 96, 915 (1999).

${ }^{4}$ O. G. Jepps, G. Ayton, and D. J. Evans, Phys. Rev. E 62, 4757 (2000).

${ }^{5}$ J. Delhommelle and D. J. Evans, J. Chem. Phys. 114, 6229 (2001).

${ }^{6}$ J. M. Simon and J. M. Rubi, J. Phys. Chem. B 115, 1422 (2011).

${ }^{7}$ A. Baranyai, Phys. Rev. E 62, 5989 (2000).

${ }^{8}$ L. Lue, O. G. Jepps, J. Delhommelle, and D. J. Evans, Mol. Phys. 100, 2387 (2002).

${ }^{9}$ F. Goujon, P. Malfreyt, A. Boutin, and A. H. Fuchs, J. Chem. Phys. 116, 8106 (2002).

${ }^{10}$ F. Goujon, P. Malfreyt, J. M. Simon, A. Boutin, B. Rousseau, and A. H. Fuchs, J. Chem. Phys. 121, 12559 (2004).

${ }^{11}$ G. Rickayzen and D. M. Heyes, J. Chem. Phys. 127, 144512 (2007).

${ }^{12}$ C. Baig and B. J. Edwards, J. Chem. Phys. 132, 184906 (2010).

${ }^{13}$ A. Lervik, Ø. Wilhelmsen, T. T. Trinh, and H. R. Nagel, J. Chem. Phys. 143, 114106 (2015).

${ }^{14}$ A. Baranyai, J. Chem. Phys. 112, 3964 (2000).

${ }^{15}$ A. Morsali, E. K. Goharshadi, G. A. Mansoori, and M. Abbaspour, Chem. Phys. 310, 11 (2005). 